\title{
Application of a carbon nanoparticle suspension for sentinel lymph node mapping in patients with early breast cancer: a retrospective cohort study
}

\author{
Liulu Zhang ${ }^{2 \dagger}$, Yijie Huang ${ }^{1,2 \dagger}$, Ciqiu Yang ${ }^{2}$, Teng Zhu², Yufeng Lin², Hongfei Gao ${ }^{2}$, Mei Yang ${ }^{2}$, Minyi Cheng ${ }^{2}$ \\ and Kun Wang ${ }^{2 *}$
}

\begin{abstract}
Background: To stage axillary lymph nodes in women with early-stage breast cancer, sentinel lymph node biopsy (SLNB), rather than axillary lymph node dissection (ALND), has been employed. Moreover, different tracer methods have various advantages and disadvantages. In recent years, carbon nanoparticle suspensions (CNSs) have been used as lymph node tracers during surgeries for thyroid cancer, gastric cancer, and colorectal cancer. The study retrospectively analyzed the feasibility and accuracy of CNS for sentinel lymph node (SLN) mapping in patients with early breast cancer.

Methods: This single-center, retrospective study included breast cancer patients who underwent SLNB from January 1, 2016, to December 31, 2017, in the Department of Breast Cancer, Guangdong General Hospital. All patients received standard SLNB surgery using a CNS tracer.

Results: A total of 332 cases were included in this study. The SLN identification rate was 99.1\% (329/332), and the mean number of SLNs was 2.6 (range, 1-6). SLN metastasis was found in 62 (18.8\%) cases, of which 90.3\% were found to be macrometastases. The sensitivity of SLNB was 95.9\% (47/49), with a specificity of 100\% (42/42), a positive predictive value of 100\% (47/47), a negative predictive value of $95.5 \%(42 / 44)$, and a false-negative rate of $4.1 \%(2 / 49)$.
\end{abstract}

Conclusion: The identification and predictive values of a CNS tracer for SLNB were satisfactory.

Keywords: Carbon nanoparticle suspension, Breast cancer, Sentinel lymph node biopsy

\section{Background}

Axillary lymph node metastasis is one of the most significant prognostic indicators of the long-term prognosis of breast cancer patients [1]. Accurate axillary staging is very important for guiding surgical treatment and choosing the appropriate adjuvant therapy. Sentinel lymph node biopsy (SLNB) has been considered the standard operation for axillary assessment in patients with early-stage breast cancer [2-4]. The traditional method for SLNB relies on the tracing process of either blue dye or radioactive colloid

\footnotetext{
* Correspondence: gzwangkun@126.com

† Liulu Zhang and Yijie Huang contributed equally to this work.

2Department of Breast Cancer, Cancer Center, Guangdong General Hospital, Guangdong Academy of Medical Sciences, Guangzhou 510080, China Full list of author information is available at the end of the article
}

or, in some cases, both [5]. The identification rate of the dual-labeled technology is $89-97 \%$, and the false-negative rate (FNR) is $5-10 \%$ [6-11].

Nevertheless, the use of radioactive colloid requires a nuclear medicine department, and there are concerns regarding the exposure of healthcare professionals to radiation [12]. Moreover, complex legislation and restrictions on the disposal and treatment of radioactive substances restrict the widespread use of radioactive colloid. In fact, in China, most patients undergo sentinel lymph node (SLN) mapping with blue dye only because radioisotope is not available at every medical center. However, blue dye-related complications, such as anaphylactic reactions, skin and fat necrosis, local inflammation, and skin staining, have been reported [13-16]. 
Due to the defects of the traditional SLN tracing method mentioned above, some new techniques have been launched in recent years. A series of studies reported the feasibility of indocyanine green fluorescence (ICG), contrast-enhanced ultrasound with microbubbles, and superparamagnetic iron oxide nanoparticles for SLN mapping in breast cancer [17]. The three methods have clinical potential to challenge the existing standard procedure, but further assessment in randomized trials is needed.

Another promising sentinel lymph node tracer is carbon nanoparticle suspensions (CNSs). With advancements in nanotechnology, CNSs could be widely used in surgeries. CNSs which contain particles with a diameter of $150 \mathrm{~nm}$ can pass much more easily through the lymphatic vessels (diameter, 120-500 nm) than through the blood capillaries (diameter, 20-50 nm). Previous studies have demonstrated the safety of CNSs [18]. In recent years, CNSs have been used as lymph node tracers during surgeries for thyroid cancer, gastric cancer, and colorectal cancer [19-21].

The aim of this study is to evaluate the accuracy and feasibility of a CNS for SLN mapping in early breast cancer retrospectively and to provide a clinical alternative technique.

\section{Methods}

\section{Patients}

This single-center, retrospective study included breast cancer patients who underwent SLNB with or without axillary lymph node dissection (ALND) from January 1, 2016, to December 31, 2017, in the Department of Breast Cancer, Guangdong General Hospital. Women with clinical T1-T3N0M0 invasive breast carcinoma were considered eligible. Exclusion criteria included inflammatory breast cancer, former surgical methods in the same axillary region, and stage IV disease. All patients received an ultrasound and a mammography to evaluate axillary lymph nodes prior to surgery. Characteristics suggestive of metastasis are not suitable for SLNB.

\section{Operative protocol}

SLNB was carried out using a 1-ml CNS (China Food and Drug Administration approval H20041829, Lai Mei Pharmaceutical Co, Chongqing, China) which was subcutaneously injected into the periareolar area. The breast was massaged for approximately $15 \mathrm{~min}$ so that the CNS was absorbed into the lymphatic duct. An incision was made into the skin and subcutaneous tissue, followed by regular separation of the skin flaps. The black-stained lymphatic duct led to the black-stained lymph nodes. After identifying the black-stained lymph nodes, during the exploration period, all black-stained lymph nodes and suspicious lymph nodes were excised (Fig. 1).

\section{Pathological examination}

Intraoperative frozen sectioning and permanent hematoxylin-eosin $(\mathrm{H} \& \mathrm{E})$ staining were performed on all lymph nodes. Macrometastasis was defined as a tumor with a diameter greater than $2 \mathrm{~mm}$, micrometastasis was defined as tumor deposition from 0.2 to $2.0 \mathrm{~mm}$, and isolated tumor cells (ITCs) were identified as a single cell or a cell cluster of no more than $0.2 \mathrm{~mm}$. A lymph node was deemed positive when a macrometastasis or a micrometastasis was determined.

\section{Statistical analysis}

Data is presented as absolute logarithms and percentages. The sensitivity (Se), specificity (Sp), positive predictive value (PPV), negative predictive value (NPV), and FNR were calculated. SPSS statistical software version 24.0 (SPSS Inc., Chicago, IL, USA) was used to manage all data.

\section{Results}

\section{Patient characteristics}

A total of 332 consecutive patients with primary breast cancer who underwent SLNB mapping with a CNS in Guangdong General Hospital were included in this study. Table 1 shows the patient characteristics. The mean age was 50 years (range, 25 to 77 years). There

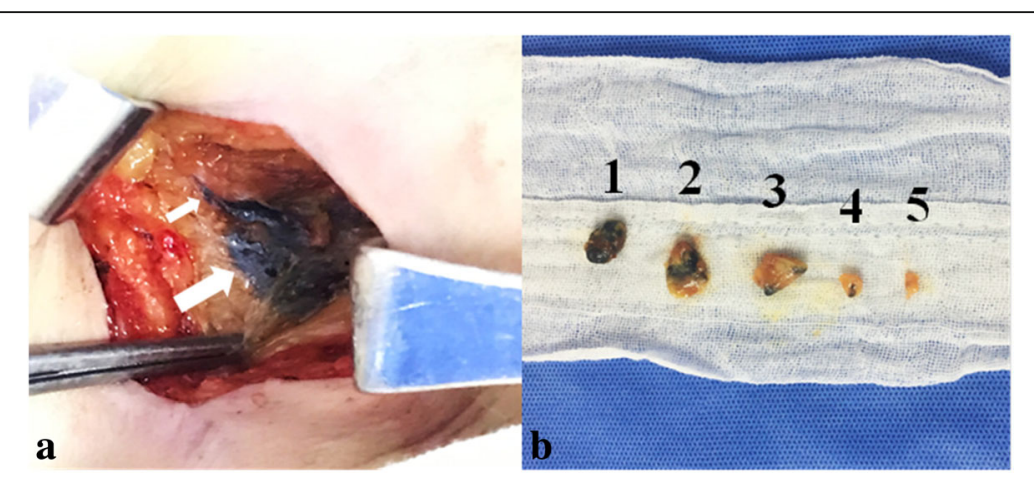

Fig. 1 Operative protocol of sentinel lymph node biopsy. a A black-stained lymphatic tract (small arrow) leads to a black-stained lymph node (big arrow). b Sentinel lymph nodes sorted by dyeing depth 
Table 1 Patient characteristics

\begin{tabular}{llll}
\hline Patient characteristics & & Number & Percentage \\
\hline Age (years) & $\geq 50$ & 155 & 46.7 \\
Tumor size & $<50$ & 177 & 53.3 \\
& T1 & 184 & 55.4 \\
& T2 & 138 & 41.6 \\
Pathology & T3 & 10 & 3.0 \\
& Invasive carcinoma & 272 & 81.9 \\
Molecular subtype & of no special type & & \\
& Other & 60 & 18.1 \\
& Luminal A & 90 & 33.3 \\
& Luminal B & 125 & 46.3 \\
& Her-2-positive & 42 & 15.6 \\
Surgery & Triple-negative & 13 & 4.8 \\
& Mastectomy & 162 & 48.8 \\
& BCS & 104 & 31.3 \\
& NSM & 66 & 19.9 \\
\hline
\end{tabular}

$B C S$ breast conserving surgery, NSM nipple-sparing mastectomy

were $184(55.4 \%)$ patients with T1, 138 (41.6\%) with T2, and $10(3.0 \%)$ with T3 tumors (according to the AJCC TNM, 7th edition). Invasive carcinoma of no special type occurred most frequently, accounting for 272 (81.9\%) patients. Molecular profiles were classified according to immunohistochemistry (IHC). Ninety (33.3\%) patients were classified as luminal A breast cancer, 125 (46.3\%) as luminal B breast cancer, $42(15.6 \%)$ as Her-2-positive type breast cancer, and $13(4.8 \%)$ as triple-negative breast cancer. Of these cases, 162 (48.8\%) underwent mastectomy, 104 (31.3\%) underwent breast-conserving surgery, and 66 (19.9\%) underwent nipple-sparing mastectomy (NSM).

\section{SLN identification}

In 329 of 332 cases, SLNs were identified by CNS. The SLN identification rate was $99.1 \%$ (329/332). There were 62 (18.8\%) cases with SLN metastases and 56 (90.3\%) macrometastases (Fig. 2). The mean number of SLNs identified was 2.6 (range, 1-6).

\section{Diagnostic value}

In total, 91 cases with ALND were included in the results of diagnostic value. In subsequent lymph node dissection, the average number of non-sentinel lymph nodes was 17.7. The pathological examination of axillary lymph nodes revealed that 42 cases were negative and 49 were positive. Forty-seven from the 49 positive cases were detected by SLNB. There were two false-negative cases that the black-stained SLNs were negative, but metastatic lymph nodes (one patient had one and another patient had three) were found in subsequent ALND. Table 2 shows the diagnostic SLNB values. Based on pathological results (the gold standard), the Se was 95.9\% (47/49), the Sp was 100\% (42/42), the PPV was $100 \%$ (47/47), the NPV was $95.5 \%$ (42/44), and the FNR was $4.1 \%(2 / 49)$.

\section{Complications of CNS use}

During and after surgery, no patient showed an anaphylactic reaction or a local inflammatory response. No patient experienced skin and fat necrosis. During the median follow-up period, which lasted 13.2 months, 139 patients (41.9\%) had skin staining (Fig. 3), most of which showed a faint black tinge, while few patients showed dark staining (Table 3).

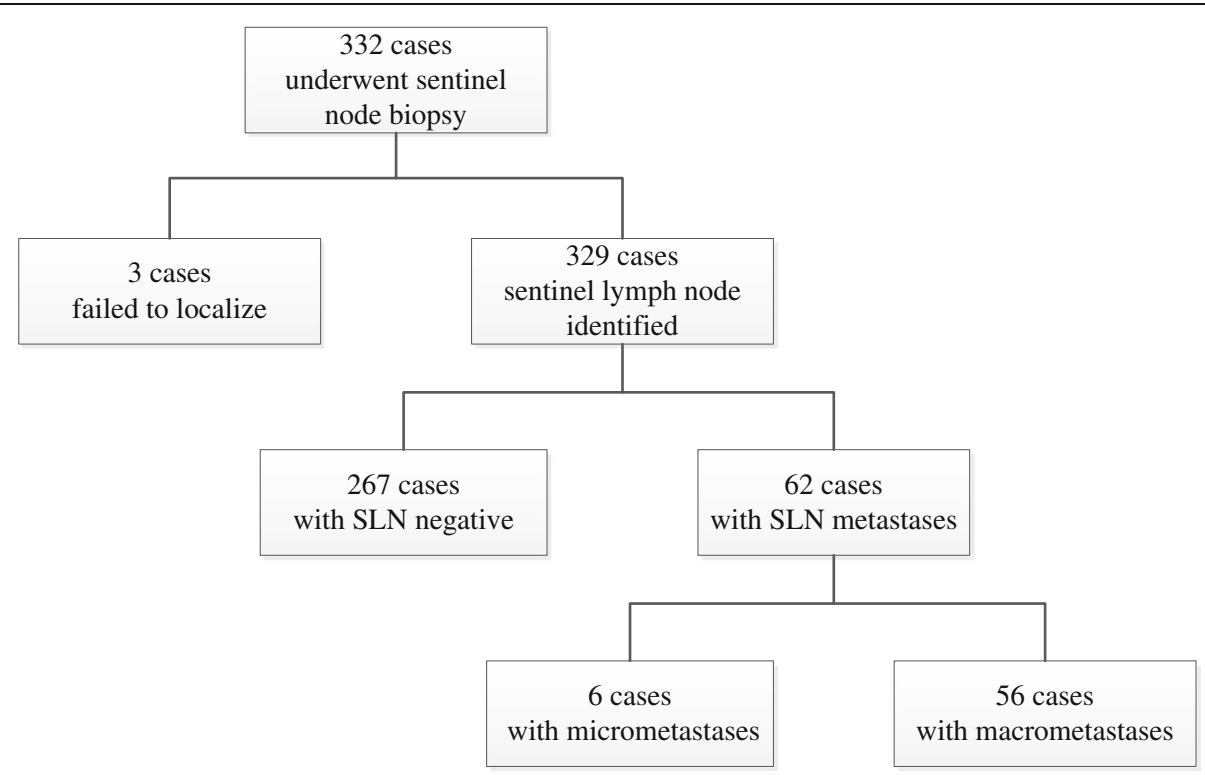

Fig. 2 Flow diagram illustrating the results of SLN identification 
Table 2 Diagnostic values of sentinel nodes using carbon nanoparticles $(n=91)$

\begin{tabular}{llllll}
\hline & & ALND & & \multicolumn{2}{c}{ Total } \\
\cline { 2 - 5 } & & Positive & Negative & & \\
\hline SLNB status & Positive & 47 & 0 & 47 & PPV $=100 \%(47 / 47)$ \\
& Negative & 2 & 42 & $44 \quad$ NPV $=95.5 \%(42 / 44)$ \\
& Total & 49 & 42 & 91 & \\
& & Se $=95.9 \%(47 / 49)$ & Sp $=100 \%(42 / 42)$ & FNR $=4.1 \%(2 / 49)$ \\
\hline
\end{tabular}

\section{Discussion}

ALND has been replaced by SLNB for early breast cancer staging of axillary lymph nodes. Blue dye, radioisotopes, and the combination are among the current mapping techniques. Because of the high identification rate, the combination of blue dye and radioactive colloid has been regarded as the gold standard of SLNB $[9,22]$. Nevertheless, the disadvantages of radioactive colloid mentioned above limit its application. However, published reports have described a high rate of identification of SLNs when blue dye only was used [23].

Some new techniques, including indocyanine green fluorescence (ICG), contrast-enhanced ultrasound using microbubbles, and superparamagnetic iron oxide nanoparticles for SLN mapping, have emerged in recent years. Compared to isotope, ICG has some advantages such as cheaper price, fewer adverse reactions, and real-time fluorescence imaging during surgery. A study including 847 women with clinical node-negative breast cancer showed that identification rate was $97.2 \%$ and the sensitivity was 95.7\% with ICG to detect SLNs [24]. A meta-analysis study illustrated that superparamagnetic iron oxide nanoparticles were non-inferior to the standard method for SLN detection that the identification rate was as high as $97.1 \%$ and the false-negative rate was $8.4 \%$ [25].

However, these techniques require intraoperative detection equipment. CNSs are relatively inexpensive, and no special equipment is required. A prospective study confirmed that CNS resulted in a comparable or better identification rate and diagnostic value than blue dye on

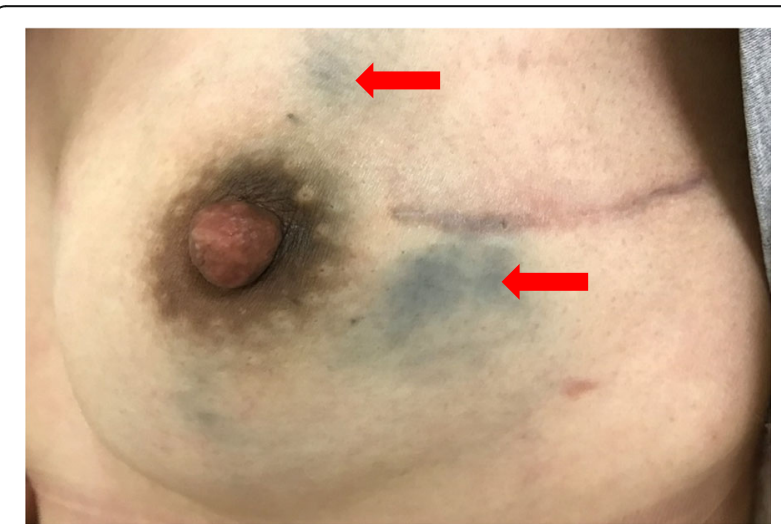

Fig. 3 Skin staining at the site of injection marking SLNs in breast cancer [26]. CNS particles have a mean diameter of $150 \mathrm{~nm}$, guaranteeing that they can pass through the lymphatic capillaries and ultimately converge in the lymph nodes which are long enough for the SLNs to be recognized during surgery. In recent years, CNSs have been employed as lymph node tracers during surgeries for thyroid cancer, gastric cancer, and colorectal cancer [19-21]. Animal experiments have demonstrated the safety of CNSs [18]. It was confirmed that CNSs can be used for SLNs mapping in patients with early-stage breast cancer, usually with success [26]. It was also determined that the best time for the maximum coloring of SLNs is 10 to $15 \mathrm{~min}$ before surgery, and the dose for a CNS of $1 \mathrm{ml}$ proved to be sufficient.

The current study was designed to retrospectively analyze not only the feasibility but also the accuracy of a CNS tracer for the SLNB procedure. In our study, the identification rate was $99.1 \%$, and the detected value was similar to or better than those reported previously [6-11]. There were three patients in whom we separated the nodes, but no black staining was observed, leading us to believe that SLNs were not successfully detected. When axillary dissection was performed, we did not detect any black-stained lymph nodes or positive lymph nodes in these patients.

Until now, the FNR remained the major evaluation criterion for the routine use of the SLNB technique. According to a meta-analysis of 69 trials, the mean FNR was 8.4\% in 8059 patients (5.5-16.7\%) [9]. When SLNB was guided by CNS tracers in our study, the FNR was $4.1 \%$, which is clinically acceptable. With a mean SLN number of 2.6 nodes in our study, the SLN number is equal to that reported in other studies that recommended using two to three SLNs to minimize the FNR $[27,28]$.

SLNB is a reliable and minimally invasive procedure. However, local and systemic complications secondary to

Table 3 CNS-related complications in sentinel node mapping $(n=332)$

\begin{tabular}{ll}
\hline Event & $n(\%)$ \\
\hline Anaphylactic reaction & 0 \\
Skin and fat necrosis & 0 \\
Local inflammation & 0 \\
Skin staining & $139(41.9 \%)$
\end{tabular}


the use of blue dye have been reported. Several studies have reported a 1 to $3 \%$ incidence of allergic and anaphylactic reactions [29]. Others have reported local skin inflammation or necrosis after blue dye injection [30]. Another drawback of blue dye is a persistent subcutaneous blue stain, with approximately $70 \%$ of patients exhibiting staining 3 months after and $41 \%$ of patients exhibiting staining 1 year after subdermal injection [14]. In our study, no patient had an anaphylactic reaction or a local inflammatory response. No patient experienced skin and fat necrosis. Skin staining seems to be the most common complication of CNS use.

However, there are still limitations in the current research. CNSs as one of the dyeing methods for SLNB, the problem of skin staining, and how to locate the black-stained lymph nodes need to be improved. Prospective studies and randomized controlled trials are required, and long-term follow-up data on the safety is still needed. In the future studies, we will use the CNS combined with another technique, which may improve the accuracy.

\section{Conclusion}

A carbon nanoparticle suspension tracer for SLNB has favorable identification rates and predictive values.

\section{Abbreviations \\ ALND: Axillary lymph node dissection; CNSs: Carbon nanoparticle suspensions; FNR: False negative rate; ICG: Indocyanine green; IHC: Immunohistochemistry; ITCs: Isolated tumor cells; NPV: Negative predictive value; NSM: Nipple sparing mastectomy; PPV: Positive predictive value; Se: Sensitivity; SLNB: Sentinel lymph node biopsy; Sp: Specificity}

\section{Availability of data and materials}

The datasets used or analyzed in the current study are available from the corresponding author on reasonable request.

\section{Authors' contributions}

KW and $L Z$ designed the study. $L Z, Y H$, and $M C$ wrote the article. $L Z, C Y$, and TZ collected and analyzed the data. $Y \mathrm{~L}, \mathrm{HG}$, and MY helped in the acquisition of the data and revised the article. KW critically revised the paper for important intellectual content and granted the final approval of the paper. All authors read and approved the final article.

\section{Ethics approval and consent to participate}

The Ethics Committee of Guangdong General Hospital approved the study. The informed consent was waived because this is a retrospective study.

\section{Competing interests}

The authors declare that they have no competing interests.

\section{Publisher's Note}

Springer Nature remains neutral with regard to jurisdictional claims in published maps and institutional affiliations.

\section{Author details}

'Department of General Surgery, Guangdong General Hospital, Guangdong Academy of Medical Sciences, Guangzhou 510080, China. ${ }^{2}$ Department of Breast Cancer, Cancer Center, Guangdong General Hospital, Guangdong Academy of Medical Sciences, Guangzhou 510080, China.
Received: 28 March 2018 Accepted: 7 June 2018

Published online: 19 June 2018

\section{References}

1. Fisher B, Bauer M, Wickerham DL, Redmond CK, Fisher ER, Cruz AB, et al. Relation of number of positive axillary nodes to the prognosis of patients with primary breast cancer. An NSABP update. Cancer. 1983;52:1551-7.

2. Lyman GH, Giuliano AE, Somerfield MR, Benson AB 3rd, Bodurka DC, Burstein $\mathrm{HJ}$, et al. American Society of Clinical Oncology guideline recommendations for sentinel lymph node biopsy in early-stage breast cancer. J Clin Oncol. 2005:23:7703-20.

3. Krag DN, Anderson SJ, Julian TB, Brown AM, Harlow SP, Costantino JP, et al. Sentinel-lymph-node resection compared with conventional axillary-lymph node dissection in clinically node-negative patients with breast cancer: overall survival findings from the NSABP B-32 randomised phase 3 trial. Lancet Oncol. 2010;11:927-33.

4. Mansel RE, Fallowfield L, Kissin M, Goyal A, Newcombe RG, Dixon JM, et al. Randomized multicenter trial of sentinel node biopsy versus standard axillary treatment in operable breast cancer: the ALMANAC trial. J Natl Cancer Inst. 2006:98:599-609.

5. Albertini JJ, Lyman GH, Cox C, Yeatman T, Balducci L, Ku N, et al. Lymphatic mapping and sentinel node biopsy in the patient with breast cancer. JAMA 1996;276:1818-22.

6. Wang L, Yu JM, Wang YS, Zuo WS, Gao Y, Fan J, et al. Preoperative lymphoscintigraphy predicts the successful identification but is not necessary in sentinel lymph nodes biopsy in breast cancer. Ann Surg Oncol. 2007;14:2215-20.

7. Goyal A, Newcombe RG, Chhabra A, Mansel RE, ALMANAC Trialists Group. Factors affecting failed localisation and false-negative rates of sentinel node biopsy in breast cancer-results of the ALMANAC validation phase. Breast Cancer Res Treat. 2006;99:203-8.

8. Krag DN, Anderson SJ, Julian TB, Brown AM, Harlow SP, Costantino JP, et al. Sentinel-lymph-node resection compared with conventional axillary-lymphnode dissection in clinically node-negative patients with breast cancer: overall survival findings from the NSABP B-32 randomised phase 3 trial. Lancet Oncol. 2010;11(10):927-33.

9. Kim T, Giuliano AE, Lyman GH. Lymphatic mapping and sentinel lymph node biopsy in early-stage breast carcinoma: a meta-analysis. Cancer. 2006; 106:4-16.

10. Straver ME, Meijnen P, van Tienhoven G, van de Velde CJ, Mansel RE, Bogaerts J, et al. Sentinel node identification rate and nodal involvement in the EORTC 10981-22023 AMAROS trial. Ann Surg Oncol. 2010;17:1854-61.

11. Zavagno G, De Salvo GL, Scalco G, Bozza F, Barutta L, Del Bianco P, et al. A randomized clinical trial on sentinel lymph node biopsy versus axillary lymph node dissection in breast cancer: results of the Sentinella/GIVOM trial. Ann Surg. 2008:247:207-13.

12. Stratmann SL, McCarty TM, Kuhn JA. Radiation safety with breast sentinel node biopsy. Am J Surg. 1999;178:454-7.

13. Zakaria S, Hoskin TL, Degnim AC. Safety and technical success of methylene blue dye for lymphatic mapping in breast cancer. Am J Surg. 2008;196:228-33.

14. Govaert GA, Oostenbroek RJ, Plaisier PW. Prolonged skin staining after intradermal use of patent blue in sentinel lymph node biopsy for breast cancer. Eur J Surg Oncol. 2005;31:373-5.

15. Stradling B, Aranha G, Gabram S. Adverse skin lesions after methylene blue injections for sentinel lymph node localization. Am J Surg. 2002;184:350-2.

16. Varghese P, Abdel-Rahman AT, Akberali S, Mostafa A, Gattuso JM, Carpenter R. Methylene blue dye - a safe and effective alternative for sentinel lymph node localization. Breast J. 2008;14:61-7.

17. Ahmed M, Purushotham AD, Douek M. Novel techniques for sentinel lymph node biopsy in breast cancer: a systematic review. Lancet Oncol. 2014;15: e351-62.

18. Xie P, Yang ST, He T, Yang S, Tang XH. Bioaccumulation and toxicity of carbon nanoparticles suspension injection in intravenously exposed mice. Int J Mol Sci. 2017;18:2562.

19. Wang LY, Li JH, Zhou X, Zheng QC, Cheng X. Clinical application of carbon nanoparticles in curative resection for colorectal carcinoma. Onco Targets Ther. 2017:10:5585-9.

20. Li Z, Ao S, Bu Z, Wu A, Wu X, Shan F, et al. Clinical study of harvesting lymph nodes with carbon nanoparticles in advanced gastric cancer: a prospective randomized trial. World J Surg Oncol. 2016;14:88. 
21. Zhao WJ, Luo H, Zhou YM, Gou ZH, Wang B, Zhu JQ. Preoperative ultrasound-guided carbon nanoparticles localization for metastatic lymph nodes in papillary thyroid carcinoma during reoperation: a retrospective cohort study. Medicine (Baltimore). 2017:96:e6285.

22. Ahmed M, Purushotham AD, Donek M. Novel techniques for sentinel lymph node biopsy in breast cancer: a systematic review. Lancet Oncol. 2014;15: e351-62.

23. Golshan M, Nakhlis F. Can methylene blue only be used in sentinel lymph node biopsy for breast cancer? Breast J. 2006;12:428-30.

24. Sugie T, Kinoshita T, Masuda N, Sawada T, Yamauchi A, Kuroi K, et al. Evaluation of the clinical utility of the ICG fluorescence method compared with the radioisotope method for sentinel lymph node biopsy in breast cancer. Ann Surg Oncol. 2016;23:44-50

25. Zada A, Peek MC, Ahmed M, Anninga B, Baker R, Kusakabe M, et al. Metaanalysis of sentinel lymph node biopsy in breast cancer using the magnetic technique. Br J Surg. 2016;103:1409-19.

26. Wu X, Lin $Q$, Chen G, Lu J, Zeng Y, Chen X, et al. Sentinel lymph node detection using carbon nanoparticles in patients with early breast cancer. PLoS One. 2015;10:e0135714.

27. Bonneau C, Bendifallah S, Reyal F, Rossi L, Rouzier R. Association of the number of sentinel lymph nodes harvested with survival in breast cancer. Eur J Surg Oncol. 2015:41:52-8.

28. Goyal A, Newcombe RG, Mansell RE. Clinical relevance of multiple sentinelnodes in patients with breast cancer. Br J Surg. 2005;92:438-42.

29. Albo D, Wayne JD, Hunt KK, Rahlfs TF, Singletary SE, Ames FC, et al. Anaphylactic reactions to isosulfan blue dye during sentinel lymph node biopsy for breast cancer. Am J Sur. 2001;182:393-8.

30. Thevarajah S, Huston TL, Simmons RM. A comparison of the adverse reactions associated with isosulfan blue versus methylene blue dye in sentinel lymph node biopsy for breast cancer. Am J Surg. 2005;189:236-9.

\section{Ready to submit your research? Choose BMC and benefit from:}

- fast, convenient online submission

- thorough peer review by experienced researchers in your field

- rapid publication on acceptance

- support for research data, including large and complex data types

- gold Open Access which fosters wider collaboration and increased citations

- maximum visibility for your research: over $100 \mathrm{M}$ website views per year 